\title{
Toma de decisiones para elegir una carrera profesional
}

\author{
Decision making in choosing a professional career
}

\author{
José Luis Alvarado Reséndiz ${ }^{1}$, Yesenia Martínez Hernánde 2 , Liliana Yadira Castellanos López ${ }^{3}$, \\ Edgar Sarabia Lugo ${ }^{4}$, Héctor Daniel Molina Rui ${ }^{5}$
}

\begin{abstract}
:
The choice of a professional career is one of the most important decisions in a person's life insofar as the life that leads to professional training is defined, and which has a direct and indirect impact on their family, work and social life. The decision can be made without concern for many young people, parents and counselors, despite the fact that they see in it the possibility and desire to improve their quality of life, education in general in the idea of professional education as the way to life or the arrival a being someone in life. From the experience as a student, a worrying panorama emerges with respect to a decision-making process to choose a professional career, for that reason it is very important the orientation in the task of lodging the student conveniently, to be able to discover their attitudes, their vocation and to choose the kind of work that you develop in your professional life. This analysis highlights the importance of guidance as the advice that should be addressed to students before starting their university and professional life.
\end{abstract}

\section{Keywords:}

Decision making, counselor, professional career

\section{Resumen:}

La elección de una carrera profesional es una de las decisiones más importante en la vida de una persona en la medida que esta definirá la vida que llevará al terminar la formación profesional, y que repercutirá directa e indirectamente en su vida familiar, laboral y social. La decisión puede ser tomada sin importancia por muchos jóvenes, padres y orientadores, a pesar de que ven en ello la posibilidad y el deseo de mejorar su calidad de vida, basados generalmente en la idea de la educación profesional como el camino para surgir o llegar a ser alguien en la vida. Desde la experiencia como estudiante, surge a la vista un panorama preocupante respecto a toma de decisiones para elegir una carrera profesional, por eso es muy importante la orientación cuya tarea es encaminar convenientemente al estudiante, para poder descubrir sus actitudes, su vocación y elegir el tipo de trabajo que desarrollará en su vida profesional. En este análisis se destaca la importancia de la orientación como del asesoramiento que debe dirigirse a los estudiantes antes de iniciar su vida universitaria y profesional.

\section{Palabras Clave:}

Toma de decisiones, consejero, carrera profesional

\section{Introducción}

La toma de decisiones para el ser humano es uno de los actos de mayor trascendencia, en virtud de elegir la forma de generar alternativas que pudiera traer consecuencias desfavorables. Hay que tener en cuenta que la toma de decisiones se hace basada en las experiencias previas, asimismo cada persona tiene una manera de afrontar la resolución de problemas, de acuerdo a sus conocimientos e historia, y los estudiantes no son la excepción para la toma de decisiones.

El desarrollo del proceso de toma de decisiones en los estudiantes para elegir la carrera profesional a cursar, al indicar las decisiones más importantes que toma el estudiante en su vida y como estas influyen en su bienestar emocional y social, al ser elecciones de peso 
que tendrán un impacto positivo o negativo. Además, el análisis de esta información hace mención de las etapas en las cuales las estudiantes toman decisiones y los personajes que influyen en este proceso como orientadores del momento que se vive, se destacan los posibles errores que puede cometer el estudiante en la toma de una decisión rápida o equivocada y qué herramientas se pueden tomar para decidir lo mejor posible.

La reflexión sobre los aspectos que el estudiante debe conocer de sí mismo para poder tomar la decisión adecuada como intereses, aptitudes y áreas o habilidades de dominio, así como el factor de motivación que influye en la elección. Además, se sugieren los puntos más sobresalientes a considerar para poder decidir la carrera universitaria del estudiante, qué herramientas le pueden servir como apoyo, a quién se puede dirigir y que factores debe de evitar para no caer en decisiones incorrectas. Además, se hace sugerencia de las carreras mejor pagadas y con mayor prestigio en México y por el contrario las carreras menores pagadas.

En el análisis documental se describen algunos conceptos de orientación educativa y se hace énfasis en su importancia, así como los personajes que están involucrados para lograr los objetivos en los jóvenes estudiantes, manifestando técnicas y estrategias que los consejeros utilizan para impartir orientación, sobre como estas actividades influyen en los alumnos para que se conozcan a sí mismos, identifiquen sus fortalezas, dominen sus miedos, utilicen sus conocimientos en la práctica, practiquen sus aptitudes y fortalezcan sus debilidades.

\section{Desarrollo}

La selección de una institución educativa para cursar una licenciatura es tal vez una de las decisiones más importantes en la vida de una persona. La toma de decisiones es una actividad que se realiza todos los días, las decisiones correctas no son fáciles de elegir, estas son el resultado de un arduo y ordenado proceso mental, por lo cual es importante no arriesgarse a una respuesta automática o un enfoque intuitivo, tomar una decisión rápida puede llevar a caer en un error o trampa. La toma de decisiones según Daft (2000, citado en Zapata, Sigala \& Miraba, 2016, p. 38) es el proceso de identificar y resolver los problemas en la organización cuando se consideran cursos alternativos de acción y se selecciona e implanta una opción. Este proceso implica una serie de hechos parciales y secuenciales que llevarán a la toma de conciencia de la necesidad de elegir, hasta elegir una solución entre las más adecuadas, tomando en cuenta la situación, y pasando por la obtención y el procesamiento de la información necesaria. Constantemente las personas toman decisiones en todo momento, de acuerdo a Solano (2003, p.49), por ser un proceso que en la mayoría de los temas, las decisiones que se toman no cuentan con información que asegure el resultado de las acciones. Por lo tanto, con el proceso lógico se reduce el número de errores, aunque todavía existe riesgo, principalmente al no se analizan a detalle todos causas que originan el problema. En el proceso se debe tomar en cuenta aspectos como el funcionamiento de los métodos, las personas implicadas y las ayudas matemáticas que puedan utilizarse.

Desde que el ser humano se encuentra en la etapa de la niñez los padres orientan a sus hijos para poder educarlos de la mejor forma, con el fin de que lleguen a una madurez plena, de igual forma cabe mencionar que las personas pasan por varios procesos de orientación como en la escuela, el trabajo la vida personal, entre otros. Cuando el joven llega al proceso de pedir ayuda para una correcta orientación vocacional busca logar su máximo desarrollo y madurez personal, citando Molina (2004 p. 6), es un proceso de asesoría al alumno en el área escolar y se expresa como eje clave en el desarrollo académico la formación de hábitos de estudio, el dominio de métodos y técnicas para el aprendizaje, y la promoción del trabajo cooperativo dentro y fuera del aula con la intervención directa de los padres y representantes.

Las personas dedicadas a la docencia especialmente en la formación de jóvenes ponen énfasis en la importancia que implica llevar una orientación que contribuya a una vida con un propósito o fin, ayudando al adolescente a recapacitar sobre sí mismo, y esperar que logre encontrar la esencia de su vida, hasta alcanzar esa libertad interior que consiste en vivir la pacífica posesión del propio camino.

Una de las etapas para tomar las decisiones, que los orientadores deben de considerar, es la obtención de información, y parafraseando a 
Canós, Pons, Valero y Maheut (2012, p. 3), en la toma de cualquier decisión se requiere primeramente obtener algún tipo de información, aunque sea muy insuficiente, y puede ser desde el perfil de ingreso y egreso de las diversas carreras profesionales. Al obtener la información se transforma, sintetizan y almacenan datos sobre un determinado suceso, porque toda información es útil antes de realizar la toma de decisiones, pero también enriquece la solución final si se incorpora pausadamente durante todo el proceso. Ciertamente, a más información, más garantía de éxito en la toma de decisiones, considerando la relación directa entre la información, su costo y el tiempo de recopilación, resumen, etc.

El orientador ha de recabar toda la información necesaria, referenciando a Mengual, Sempere, Juárez, y Rodríguez (2012, p. 8), para conocer cuál es el problema y sus causas de para elegir una carrera profesional, con el propósito de realizar una puntual definición del problema. Para recabar información se recomienda: preguntar a personas relacionadas directamente con el problema, ir a la zona o área donde se está produciendo o se ha producido el problema, y solicitar información a expertos en el lugar competente. Hay que tener en cuenta que no siempre la información obtenida es fehaciente ni completa al $100 \%$, porque en ocasiones la gente proporciona su interpretación de la información obtenida y omite parte de la misma. Es importante preguntar a distintas personas y cruzar la información recibida, filtrarla y comprobar, para atribuir si es la necesaria para orientar a estudiantes en la toma de decisiones al momento de realizar la elección de su carrera profesional.

La calidad del proceso mismo de elección profesional para González (2009, citado en Vásquez, 2010, p. 4), lo compone una de las bases de la elección responsable y considera como determinante la posición que toma el estudiante en el proceso de elegir su carrera profesión. Cuando la profesión se elige responsablemente, el sujeto asume una postura personal, sensata y activa en su elección, hay mayor posibilidad de éxito en el trayecto de estudiar su carrera. Por lo tanto, se consideran dos elementos esenciales en la competencia para la elección profesional responsable: la autodeterminación y la conducta exploratoria.

La orientación vocacional es una estructuración de propuestas en torno al trabajo de las instituciones educativas y las familias de los estudiantes, por eso razón se convierte en una herramienta para los estudiantes en la toma de decisiones. Por su parte Reyes y Novoa (2014, p. 14) la exploración vocacional adquiere una especial importancia, por la observación de manifestaciones constantes en gustos, intereses y habilidades desde la cotidianidad a edades tempranas proporciona herramientas valiosas para la construcción de modelos metodológicos de intervención, que consoliden habilidades y competencias, para un futuro desempeño ocupacional o profesional.

En la educación técnico-profesional como parte de la orientación vocacional tiene como propósito de capacitar al estudiante en el desarrollo de una determinada carrera con la utilizando de los conocimientos teóricos y habilidades prácticas. Así se aportaría una formación profesional entre el sistema educativo y el mundo del trabajo. Hoy en día los cambios sociales reclaman un sistema de formación profesional con los siguientes requisitos: primeramente la flexibilidad para adaptarse a las necesidades y requerimientos del sistema productivo, agilidad y capacidad de respuesta al desafío del cambio tecnológico y del mercado de trabajo, capacidad de promocionar a las personas proporcionando los elementos educativos de carácter adecuado, que permitan hacer frente a las distintas demandas laborales, de igual forma a los avances en su formación y cualificación, autorregulación mediante mecanismos que respondan la actualización y renovación del sistema en objetivos, contenidos y métodos.

La indagación sobre las posibilidades educativas y ocupacionales, como lo menciona Mosca y Santiviago (2006, p. 19), es fundamental para tomar cualquier decisión respecto al futuro vocacional. Es la materia prima para pensar y decidir sobre las diferentes opciones de futuro. Es evidente que sin información no se puede elegir 
con facilidad la elección de una carrera, lo que es claro también, es que la información es necesaria por ser la etapa básica para recorrer, pero no es suficiente en un proceso de elección de su carrera profesional.

Al hablar de niveles de actuación en la orientación educativa, la tutoría es una actividad implícita en la labor docente que se realiza individual 0 colectivamente con los estudiantes de un grupo o clase, con el propósito de favorecer la unificación de los procesos de aprendizaje y un desarrollo del sujeto en lo cognitivo, afectivo y social, motivo que lleva a el departamento de orientación a encargarse de la ubicación de todo, razón por la que deberá de estar constituido por especialistas en el campo psicopedagógico.

En concordancia con lo expuesto Álvarez (1995, citado en Ureña y Barboza 2015, p. 3), al referirse a la orientación vocacional también como un proceso de desarrollo de la carrera a lo largo de la vida de las personas, principalmente a los estudiantes para elegir una carrera a estudiar, y al mismo tiempo afirma a que está presente en los diferentes contextos en que se encuentren éstas, por ejemplo:

- El educativo que abarca el período formativo, desde la preparación para la vida laboral, que se desarrolla fundamentalmente en el contexto escolar y comunal.

- Dentro de este contexto la orientación vocacional desarrolla diferentes acciones con el estudiantado, padres, madres, personas encargadas y docentes con el propósito de favorecer el desarrollo integral del estudiante y al mismo tiempo el vocacional.

- $\quad$ El organizacional que incluye el período de desempeño laboral que tiene lugar en el centro de trabajo, donde se dan gran parte de las experiencias de las personas relacionadas necesariamente con el trabajo, cumpliendo una doble función, tanto a nivel individual como social. - $\quad$ El tiempo libre, que tiene que ver con el tiempo que comparte la persona con el trabajo o en el período de retiro laboral, al creer que se cuenta con más tiempo disponible para realizar aquellas actividades que no se han logrado ejecutar antes, a pesar de que se quería llevar a cabo, pero que por alguna situación se postergó, el poder hacerlo.

Para que los jóvenes tomen una decisión acertada se necesita elaborar un plan de actuación que indique los objetivos laborales, por ejemplo: qué desea alcanzar el estudiante, qué áreas profesionales son de su interés, identificar las diversas alternativas, las consecuencias positivas y negativas, anticipar las consecuencias de llevar a cabo estas alternativas en sus diferentes momentos como a corto, medio y largo plazo. Es necesario tener presente los costos económicos, esfuerzo, tiempo, abandono de otras actividades, etc., así mismo tener en cuenta los apoyos, eliminar alternativas no interesantes 0 no convenientes y finalmente hacer la elección de la opción o alternativa.

Los estudiantes al momento de preferir la carrera profesional a estudiar caen en errores o tienen duda acerca de cuál será la trayectoria apropiada a seguir, por ellos la orientación vocacional juega un rol importante ya que posibilita al estudiante herramientas para poder tomar la decisión correcta. La persona encargada de orientar tiene la responsabilidad no sólo de ubicar en un área específica al estudiante, sino de capacitarlo para que manipule con instrumentos eficientes, un mundo interno y profesional cada día más cambiante y complejo.

La persona encargada de orientar a los jóvenes deberá, según International Youth Foundation (2014), implementar una serie de sesiones y actividades interactivas que se pueden llevar a cabo con los jóvenes en la orientación de la planificación del desarrollo laboral y a tomar medidas necesarias para alcanzar sus metas laborales. El orientador vocacional tiene un trabajo complejo debe de facilitar el aprendizaje de destrezas del alumno con actividades que le interesen y pueda dominar, influyen las creencias que le ha inculcado la familia, los valores que practica, los hábitos de trabajo y cualidades personales, por eso el orientador necesita conocer a cada alumno y ayudarle a crear una vida placentera en un mundo laboral constantemente cambiante. 
La orientación debe tener como principal fin el de descubrir el potencial de cada persona y lograr que cada uno desarrolle sus capacidades al máximo para que pueda ofrecer lo mejor así mismo y a los demás., se puede definir como un proceso que implica acciones para ayudar a los estudiantes en la solución de problemas críticos y conflictivos, así como lograr la satisfacción de necesidades para el logro de un bienestar agradable con lo que se realiza. Las instituciones de educación superior, citando a Reyes y Novoa (2014, p. 16), deben organizar eventos sobre la importancia de continuar estudios profesionales. Este tipo de estrategia se convierte en una herramienta enriquecedora para los estudiantes, pues adquieren elementos de juicio para la toma de decisiones sobre su formación profesional

El estudiante también necesita de otras personas que lo orienten y apoyen en la preparación de su proyecto de vida, en donde acumulará información tanto interna como externa para tomar decisiones. Debe aprender a reconocer sus intereses, aptitudes y áreas universitarias dominantes, de manera que cuente con elementos necesarios para una elección vocacional. Al elegir un futuro se convierte en un desafío dudoso e inquietante, mucho más cuando la persona tiene una edad joven, ya que sus intereses 0 prioridades pueden ser otros aspectos. La orientación vocacional le debe brindar al estudiante dos direcciones: por un lado, ayuda al alumno al conocimiento de su persona y por otra parte ayuda a conocer las ofertas educativas que las instituciones ofrecen, así como en qué lugares se pueden incorporar en el mercado laboral. Además, la familia influye en esta decisión pues los padres esperan un hijo exitoso, pero no sólo importa lo que esperen de él, sino que también todo lo que haga sea hecho con pasión, amor a la profesión, creatividad en lo que se realiza y entusiasmo por hacer las cosas.

La orientación vocacional en la educación de nivel medio superior en el siglo XXI, de acuerdo a Bonilla, López y Juárez (2015), está enfocada en nuevas estrategias metodológicas para mejorar el aprendizaje, de igual manera promueve y ayuda a que los estudiantes elijan adecuadamente su vocación real, al mismo tiempo que aporta a que las condiciones del sistema tanto político, económico y social sean más humanas y favorecedoras para la sociedad. Los estudiantes enfrentan una de las decisiones más importantes en su vida, la de cómo elegir la carrera universitaria, elegir la profesión a la que se dedicarán requiere reflexionar sobre qué es lo que más les gustaría hacer en un futuro, es necesario analizar que habilidades se poseen para proyectar esas expectativas, va más allá de conocer las materias de la carrera y saber si son interesantes o si el estudiante puede ser capaz de dominarlas, pues se trata de entender que esa decisión tendrá como consecuencia el desempeño profesional y el futuro laboral.

Para tomar la decisiones de elegir una carrera profesional, es importante conocer las opciones profesionales que se consideran según afinidades y capacidades más sobresalientes de la persona, se debe tener en claro de qué se trata, qué perfil ocupacional tiene cada opción, cuáles son las especialidades que se pueden cursar posteriormente y si el estudiante está dispuesto a seguir sus estudios según lo requiera la carrera, teniendo en claro que la educación es permanente y en esta época exige seguir preparando a los profesionales. Hay ocasiones que los estudiantes carecen de apoyo para tomar este tipo de decisiones, aún más si se dejan guiar por el tiempo, es decir caen en el error de empezar sus estudios lo antes posible para poder terminar e incorporarse en el mercado laboral siendo muy jóvenes, esto no siempre es positivo ni lo más indicado, las empresas requieren cierta edad y experiencia para poder laborar.

La elección de una profesión, citando a Caldas y Hidalgo (2018, p.6), debe ser una decisión meditada, en virtud de marca la vida de una persona y su sentimiento de satisfacción como profesionista. Debe de tener en cuenta que la vida laboral y la vida personal deben ir en concordancia y en la misma dirección. De igual forma los autores proponen que para tomar una decisión es conveniente que se analicen los siguientes aspectos: 
- El potencial profesional, los intereses personales, las fortalezas y las debilidades propias

- Los diferentes caminos profesionales que se pueden elegir, las capacidades, competencias y actitudes que requieren las ocupaciones que les interesan.

- El objetivo profesional y su proyecto profesional

- $\quad$ El mercado laboral del que va a formar parte. Una vez que se ha profundizado en el autoconocimiento, es necesario que conozcan las realidades del mercado laboral y como se accede a él.

- Trazar un plan de acción en el que se relaten los pasos que hay que dar para lograr los objetivos establecidos.

En la decisión de una carrera profesional es necesario el aprendizaje profesional; razón que destaca la importancia de la orientación cuya labor es encausar convenientemente al futuro estudiante universitario, para que le permita descubrir sus actitudes, determinar su vocación y escoger el tipo de trabajo que desempañara en su vida profesional. El asesoramiento debe dirigirse a los estudiantes para toma la decisión de elegir una carrera profesional, antes de iniciar una vida universitaria. La orientación y formación profesional son dependientes entre sí, la primera debe contar con las posibilidades del aprendizaje, y la segunda con aspirantes idóneos para ejercer su profesión, ambas características sirven al mismo propósito, en donde los jóvenes estudiantes estén satisfechos con la profesión elegida y desempeñarse correctamente. Cuando el estudiante elige una carrera acertadamente, alcanza la satisfacción para sí mismo y para la sociedad, al cumplir los objetivos establecidos.

Sin embargo, es bastante frecuente que el estudiante no cuente con la suficiente madurez para la labor de elegir una carrera profesional, al ser una persona que carece de experiencia y tiene limitaciones, al mismo tiempo obstáculos para una elección de una carrera profesional idónea, por ejemplo: la inestabilidad de su propia edad, considerando que esta inquietud generalmente se presenta en la adolescencia, al considerarse un período de transición entre la niñez dependiente y la edad adulta independiente, razones que permiten desatacar la importancia de la orientación para facilitar al estudiante en toma una decisión para elegir una carrera profesional.

\section{Conclusiones}

Una de las decisiones más importantes en la vida de las estudiantes es tomar la decisión de elegir una carrera profesional, es evidente que se pasará gran parte del tiempo en el trabajo y al desarrollo de la profesión, además continuar estudiando la carrera que se desea ayuda al cumplimiento de los sueños o metas desde el ámbito personal y laboral. Los jóvenes que actualmente cursan una carrera universitaria en México no tomaron una decisión informada desconocen si esa profesión les será rentable en el futuro., caen en el error de tomar decisiones basadas en la popularidad de las carreras, porque son las que conocen o porque sus familiares y amigos que conocen estudiaron esto sin embargo esto no puede ser la mejor manera de elegir.

En la etapa de la vida de los jóvenes en que tienen que tomar decisión para elegir una carrera profesional, se encuentran las emociones que originan modificaciones internas, como la inseguridad, en donde el estudiante muchas veces se siente emocionalmente inseguro y más aún le toca decidirse por alguna carrera a estudiar, generando una serie de dudas. El estudiante es inestable como la víctima de un estado indefinido y caótico de emociones contradictorias. Además la elección de carrera profesional es difícil, porque se encuentra desplazado a la realidad tanto del mundo que lo rodea como también de las posibilidades o limitaciones personales que posee para encaminarse hacia una decisión acertada.

El tomar la decisión de elegir una carrera profesional, el presente y el futuro no solo de la persona sino también de su familia y del contexto social donde se desenvuelve, de manera que en su presente forma una inversión de tiempo y dinero, que indudablemente le puede sumar o restar a su condición socioeconómica y/o la de su familia. El futuro representará la calidad de vida en términos de comodidad, plenitud, felicidad, 
infelicidad o inconformidad, y de acuerdo a Rauduvaitè y Lasauskienè (2013, citado en Bravo y Vergara, 2018, p. 44), al comienzo de la planificación de la carrera profesional, a menudo tienen lugar consideraciones sobre la profesión en la que una persona se encuentra mejor, y aun tomando las decisiones nada asegura que la vida profesional será plena y satisfactoria, el alumno debe tomarse tiempo para reflexionar y evaluar todas las opciones posibles, esto conduce a reducir el riesgo de equivocarse y aumenta la posibilidad de alcanzar el éxito deseado.

La falta de una completa orientación profesional permite que ocurra la consecuencia de no identificar si poseen las capacidades o habilidades necesarias para desempeñar determinada profesión y facilitar la tomar de decisión. Debido a que en la mayoría de los casos los orientadores no profundizan realmente en las vocaciones profesionales de los estudiantes, es decir, el proceso de orientación vocacional es muy importante al momento de tomar la decisión de elegir una carrera profesional.

Tomar la decisión de elegir una carrera profesional, implica hacer tiempo sobre los diversos factores que intervienen y las distintas posibilidades que se tienen para elegir una carrera profesional, es decir, requiere de tomar una postura personal, activa y consciente, por parte del estudiante al momento de elegir. Finalmente, el proceso de toma de decisiones requiere momentos guiados de verbalización y análisis que posibiliten la consideración de todos los elementos que una decisión implica, labor en que se encuentra implicado el orientador para facilitar al estudiante la toma de decisiones.

\section{Bibliografía}

Bravo Torres G. y Vergara Tamayo M. A. (2018). Factores que determinan la elección de carrera profesional: en estudiantes de undécimo grado de colegios públicos y privados de Barrancabermeja. El V. 12, N 20 de la revista Psicoespacios. Recuperado de: https://dialnet.unirioja.es/buscar/documentos?qu erysDismax.DOCUMENTAL_TODO=elecci\%C3 $\% \mathrm{~B} 3 \mathrm{n}+\mathrm{de}+$ una+carrera+universitaria\&registros $\mathrm{P}$ orPagina $=20 \&$ camposOrdenacion $=\% 7 B D O C U M$ ENTAL_SORT_ANYOPUB\%3DDESC\%7D
Bonilla Bonilla L., López Castro S. L., y Juárez Hernández A. (2015). El Impacto De La Orientación Vocacional En Los Estudiantes De $5^{\text {to }}$ y $6^{\text {to }}$ Semestre Del Cecyte 02 De Xicohtzinco. Recuperado de: https://posgradoeducacionuatx.org/pdf2015/E051 .pdf

Caldas Blanco M. E., y Hidalgo Ortega M. L. (2018). Formación y orientación laboral 360․ Editex. Recuperado de: https://books.google.com.mx/books?id=DhlfDwA AQBAJ\&pg=PA6\&lpg=PA6\&dq=pasos + para+ele gir+una+carrera+profesional+2013..2018\&source =bl\&ots=rHYp3a3zXo\&sig=8bIEMXrgh1yWQa1o Ozhjah2zgHo\&hl=es-

419\&sa $=X \& v e d=0$ ahUKEwiqn9y9KvcAhWina0KHbG1Dv0Q6AEIZjAK\#v=onepag e\&q\&f=false

Canós Darós L., Pons Morera C., Valero Herrera M. y Maheut J. P. (2012). Toma de decisiones en la empresa: proceso y clasificación. Departamento Organización de Empresas. Universidad Politécnica de Valencia. Recuperado de: https://riunet.upv.es/bitstream/handle/10251/165 02/TomaDecisiones.pdf

International Youth Foundation (2014). Descubre Tu Vocación. Planes de sesión y actividades para facilitar la orientación vocacional. Recuperado de: https://www.jovenesneo.org/Portals/13/lmages/N EO/SW_Recursos/8db87891-3e82-4466-b5dae314f9e0025e_Manual\%20\%20Descubre\%20tu\%20vocacion.pdf

Mengual Recuerda A., Sempere Ripoll F., Juárez Varón D. y Rodríguez Villalobos A. (2012). El Proceso De Toma De Decisiones Como Habilidad Directiva. Revista de investigación. Editada por Área de Innovación y Desarrollo, S.L. Recuperado de: https://www.3ciencias.com/wpcontent/uploads/2012/07/4.El-proceso-de-tomade-decisiones.pdf

Molina Contreras D. L. (2004). Concepto De Orientación Educativa: Diversidad $Y$ Aproximación. Revista Iberoamericana de Educación (ISSN: 1681-5653). Recuperado de: https://rieoei.org/historico/deloslectores/736Molin a108.PDF

Mosca De Mori A., y Santiviago C. (2006).Conceptos y herramientas para aportar a la orientación vocacional ocupacional de los jóvenes. Programa de Respaldo al Aprendizaje de la Comisión Sectorial de Enseñanza de la Universidad de la República. Recuperado de: www2.compromisoeducativo.edu.uy/.../MANUAL -CONCEPTOS-Y-HERRAMIENTA...

Reyes Campos I. M., y Novoa Cely A. M. (2014). Orientación Vocacional. Publicación Resultado Del Convenio 3351 Suscrito Entre La Secretaría De Educación Distrital Y La Universidad Central. 
ISBN: 978-958-26-0224-6. Primera edición. Impreso en Colombia-Printed.

Solano, Ana Isabel (2003). Toma de decisiones gerenciales. Tecnología en Marcha. Vol. $16 \mathrm{~N}^{\circ} 3$. Instituto Tecnológico de Costa Rica. Recuperado de:

https://dialnet.unirioja.es/descarga/articulo/48357 19.pdf

Ureña Salazar V., y Barboza Arrieta C. (2015). Aportes De La Orientación Vocacional En El Contexto Laboral. Revista Electrónica Actualidades Investigativas en Educación, vol. 15, núm. 1, pp. 1-21. Universidad de Costa Rica. San Pedro de Montes de Oca, Costa Rica. Recuperado de: http://www.redalyc.org/pdf/447/44733027040.pdf

Vásquez Carmona J. (2010). El Proceso De Toma De Decisiones. Acompañamiento Formativo a Estudiantes de Educación Media en el Proceso de Elección de Estudios Superiores. Ministerio de Educación Nacional - Universidad de Antioquia. Recuperado de: http://aprendeenlinea.udea.edu.co//ms/men_ude a/pluginfile.php/29949/mod_resource/content/0/ modulo_4/pdfs/mod04_doc01_decisiones.pdf

Zapata Rotundo G.J., Sigala Paparella L., Mirabal Martínez A. (2016). Toma De Decisiones Y Estilo De Liderazgo: Estudio En Medianas Empresas. Compendium, vol. 19, núm. 36, pp. 35-59. Universidad Centroccidental Lisandro Alvarado Barquisimeto, Venezuela. Recuperado de: http://www.redalyc.org/pdf/880/88046587003.pdf 